\title{
Arrested Plasmodium liver stages as experimental anti-malaria vaccines
}

\author{
Kai Matuschewski, ${ }^{1, *}$ Julius Clemence Hafalla, ${ }^{2}$ Steffen Borrmann ${ }^{3,4}$ and Johannes Friesen ${ }^{1}$
}

'Parasitology Unit; Max Planck Institute for Infection Biology; Berlin, Germany; ${ }^{2}$ Department of Immunology and Infection; Faculty of Infectious and Tropical Diseases; London School of Tropical Medicine and Hygiene; London, UK; ${ }^{3}$ Department of Infectious Diseases; Heidelberg University School of Medicine; Heidelberg, Germany;

${ }^{4}$ Kenya Medical Research Institute; Wellcome Trust Research Programme; Kilifi, Kenya

Key words: malaria, Plasmodium, sporozoite, whole organism vaccine

Abbreviations: Abx, antibiotics; AZ, azithromycin; CSP, circumsporozoite protein; CQ, chloroquine; $\boldsymbol{\gamma}$-spz., irradiated sporozoites; GAPs, genetically arrested parasites; hk spz., heat killed sporozoites; PQ, primaquine;

SE/DC, sporozoite exposure under drug cover; spz., sporozoites

Eukaryotic pathogens typically follow a complex life cycle, including host switch and morphologically distinct forms. Parasite stage conversion offers exceptional opportunities for whole organism vaccine development. In the case of Plasmodium, the causative agent of malaria, disease is exclusively caused by asexual blood stages that invade and replicate within erythrocytes. Pathogenic blood stage infections are preceded by a silent parasite growth phase inside the liver. Two alternative experimental whole organisms vaccine strategies that lead to arrested Plasmodium liver stages elicit potent, lasting immunity against re-infection. Live irradiation- or genetically arrested parasites are metabolically active and correspond to classical attenuated vaccines. Specific antimalarial treatment during experimental natural sporozoite infections prevents a febrile malaria episode and, simultaneously, induces effective anti-liver stage immunity. Translation of these strategies into a safe, affordable and accessible pediatric anti-malaria vaccine requires major bioengineering and pharmaceutical improvements, respectively, but holds promise for a truly effective immunization scheme against the most prevalent and fatal vector-borne disease.

\section{Introduction}

Malaria is caused by unicellular, obligate intracellular eukaryotes of the genus Plasmodium that can invade and replicate within erythrocytes. This vector-borne parasitic disease continues to pose a major disease burden and death toll, primarily in infants and children living in Sub-Saharan Africa. ${ }^{1}$ As in all apicomplexan parasites, the pathogen life cycle follows a complex developmental program, in the case of Plasmodium inside the mosquito and mammalian hosts. The signature febrile episodes are caused by the synchronized rupture and re-invasion

*Correspondence to: Kai Matuschewski;

Email: matuschewski@mpiib-berlin.mpg.de

Submitted: 11/16/10; Accepted: 11/20/10

DOI: $10.4161 /$ hv.7.0.14557 of the host erythrocytes. Exponential expansion of the parasite population during asexual blood stage growth leads to malariarelated morbidity and clinical symptoms. A proportion of clinical malaria cases develop life-threatening complication, which can include anemia, multi-organ failure or cerebral malaria. ${ }^{2}$ What triggers disease exacerbation remains an open question. It is believed that contributing factors include the initial sporozoite dose inoculated during the mosquito bite, parasite virulence, host genetics and co-infections with helminths or bacteria..$^{3-7}$ Reliable biomarkers that would predict disease progression are still missing.

In endemic areas anti-malaria immunity is only acquired gradually after many repeated exposures. ${ }^{8,9}$ The first responses to develop in children protect against severe complications without affecting mild disease or parasite burden. Over time, often not before adolescence, anti-disease immunity is mounted, leading to 'clinical tolerance' as opposed to sterilizing immunity. Field studies also show that antibody responses are short-lived and necessitate continuous re-exposure to eventually persist..$^{10,11}$ A proportion of adults remains symptom-free and parasitepositive, and hence, do not receive treatments, contributing to the continuous parasite propagation via mosquito transmission. Together, naturally acquired anti-malaria immunity is slow, incomplete, short-lived and strain-specific. Whether immunization strategies can mount more potent and lasting protective immune responses and surpass nature remains to be shown.

Before the onset of a blood stage infection Plasmodium undergoes an obligate, yet diagnostically and clinically silent, population expansion phase in the liver. ${ }^{12,13}$ Intra-hepatic development compensates for one of the two bottlenecks, i.e., sporozoite inoculation during the short probing phase of a mosquito bite, in the Plasmodium life cycle and leads to formation of thousands of infectious merozoites. Sporozoite-to-merozoite stage conversion takes several days and represents a unique window of opportunity for anti-malaria vaccine development. ${ }^{14}$ Recent experimental whole organism vaccine approaches in rodent malaria systems have consistently demonstrated long-lasting sterilizing immunity against reinfection. Complementary vaccine approaches aim at targeting the pathogenic blood stages and transmission stages..$^{15-18}$ 


\begin{tabular}{|c|c|c|c|c|c|}
\hline $\begin{array}{l}\text { sporozoite } \\
\text { malaria } \\
\text { vaccine }\end{array}$ & $\begin{array}{l}\text { transmigration } \\
\& \text { invasion }\end{array}$ & $\begin{array}{l}\text { initial } \\
\text { development }\end{array}$ & $\begin{array}{l}\text { further } \\
\text { development }\end{array}$ & $\begin{array}{l}\text { principle of } \\
\text { attenuation }\end{array}$ & $\begin{array}{l}\text { protection } \\
\text { against } \\
\text { reinfection }\end{array}$ \\
\hline $\begin{array}{l}\text { - heat-killed } \\
\text { sporozoites } \\
\text { (hk spz) }\end{array}$ & & & & $\begin{array}{l}\text { killed } \\
\text { parasites }\end{array}$ & - \\
\hline $\begin{array}{l}\text { - irradiated } \\
\text { sporozoites } \\
(\gamma \text {-spz) }\end{array}$ & & & & $\begin{array}{l}\text { DNA strand } \\
\text { breaks }\end{array}$ & + \\
\hline $\begin{array}{l}\text {-genetically } \\
\text { arrested } \\
\text { parasites (GAPs) }\end{array}$ & & & & $\begin{array}{l}\text { Genetically } \\
\text { defined arrest }\end{array}$ & + \\
\hline $\begin{array}{l}\text { - Sporozoites + } \\
\text { chloroquine cover } \\
\text { (spz/CQ) }\end{array}$ & & & & $\begin{array}{l}\text { suppressive } \\
\text { treatment }\end{array}$ & + \\
\hline $\begin{array}{l}\text { - Sporozoites + } \\
\text { primaquine cover } \\
\text { (spz/PQ) }\end{array}$ & & & & $\begin{array}{l}\text { causal } \\
\text { prophylaxis }\end{array}$ & + \\
\hline $\begin{array}{l}\text { - Sporozoites + } \\
\text { azithromycin cover } \\
\text { (spz/AZ) }\end{array}$ & & & & $\begin{array}{l}\text { delayed } \\
\text { parasite death }\end{array}$ & + \\
\hline
\end{tabular}

Figure 1. Experimental approaches for developmental Plasmodium liver stage arrests. Heat-killed sporozoites (hk spz) cannot enter a suitable hepatocyte and, hence, fail to elicit protection against re-infection, indicative of a minor role of antibodies against sporozoite surface proteins in lasting protective immunity. Irradiated sporozoites $(\gamma$-spz) retain their capacity to actively enter their host cells and initiate the transformation process inside a hepatocyte. They are replication-deficient because of multiple random DNA double strand breaks but persist as metabolically active parasites and elicit lasting protection. Genetically arrested parasites (GAPs) contain tailor-made, stable deletions of Plasmodium genes that exert vital functions during liver stage development. Similar to $\gamma$-spz, GAPs persist as metabolically active parasites and confer protection. Experimental sporozoite exposure (SE) under drug cover (DC) aims at preventing febrile malaria while simultaneously inducing pre-erythrocytic immune responses. SE under chloroquine cover (spz + CQ) can induce brief, but mild malaria episodes. In addition, global CQ resistance necessitates inoculation of CQ-sensitive laboratory strains under clinical surveillance. Sporozoite exposure under primaquine cover (spz + PQ) takes advantage of a causal-prophylactic drug, which kills intra-hepatic parasites. Intracellular killing by pharmacological treatment induces lasting protection. Sporozoite exposure under azithromycin cover (spz $+\mathrm{AZ})$, a safe antibiotic licensed for children and during pregnancy, induces a delayed parasite death, leading to complete maturation of liver stages, yet inviable liver stage merozoites. This strategy combines the advantages of drug cover by CQ, i.e. full maturation and, hence, maximum antigen display and $P Q$, i.e. global parasite susceptibility to the drug and prevention of blood stage infections and malaria symptoms during the immunization phase.

Here, we present an overview of the different strategies that lead to a pre-erythrocytic life cycle arrest.

\section{Radiation-Attenuated Sporozoites: First Generation Whole Organism Vaccines}

Malaria vaccinology started with immunizations in experimental animals using killed blood stage parasites plus adjuvants ${ }^{19,20}$ and killed or UV-inactivated sporozoites ${ }^{21,22}$ or both parasite stages. ${ }^{23}$ These traditional whole organism vaccine strategies offered only partial, if any, protection against re-infection. The findings established that eliciting anti-sporozoite or -blood stage immune responses, at least by the approaches available at that time, do not offer a path towards malaria vaccine development. Moreover, the experiments already indicated that vaccine strategies against a complex, slow-growing eukaryotic pathogen need to be fundamentally different from anti-viral vaccines, which often mimic naturally acquired immunity.

The first conclusive demonstration that lasting protection can be elicited by a whole organism vaccine strategy was by a series of immunization studies performed at New York University using intravenous injection of radiation-attenuated sporozoites $(\gamma$-spz or RAS) in mice ${ }^{24-30}$ (Fig. 1). These findings established that sporozoites need to be viable in order to confer protection. Heatkilled sporozoites (hk spz), for instance, cannot enter a suitable hepatocyte and, hence, fail to elicit protection against re-infec$\operatorname{tion}^{31}$ (Fig. 1). Moreover, protection following $\gamma$-spz immunization is acting exclusively against the pre-erythrocytic stages, since bypassing the life cycle by transfusion of infected blood led to a fulminant malaria infection. Subsequent work in rodent models established that protective immunity is of multifactorial nature and directed against free sporozoites through high titers of blocking antibodies and intracellular liver stages via IFN $\gamma$ secreting conventional $\alpha \beta$ T cells, as well as NK cells and $\gamma \delta$ T cells. ${ }^{13,32-34}$ The $\gamma$-spz immunization strategy has been swiftly translated to trials in non-human primates ${ }^{35}$ and human volunteers. ${ }^{36}$

The demonstration of lasting sterile protection in a number of vaccinees that received a high number of bites from irradiated $P$. falciparum-infected Anopheles mosquitoes ${ }^{36}$ has been confirmed in a number of subsequent small phase IIa trials and established the current gold standard for an anti-malaria vaccine. $^{37,38}$ It has been argued that the translation of $\gamma$-spz from an 
experimental vaccine to a pediatric formulation is hampered by several hurdles and, hence, impractical. ${ }^{39}$ Vaccination with $\gamma$-spz, however, most likely induces very potent, strain transcending long lasting cellular immune responses. It remains to be formally demonstrated that a subunit vaccine can achieve lasting protection against a eukaryotic pathogen. Delivery of the gold standard vaccine to the people in need is an ambitious goal that deserves sustained commitment from researchers, funding agencies and public health authorities. Towards this goal, researchers currently explore practical and efficient vaccine administration routes, sporozoite purification and freezing methods and logistics to distribute liquid nitrogen-frozen vials to rural health centers. ${ }^{40,41}$

An alternative vaccine candidate that emerged from the studies utilizing $\gamma$-spz was the successful cloning of the major $P$. falciparum sporozoite surface protein, termed circumsporozoite protein (CSP), ${ }^{42}$ and the subsequent development of the RTS,S subunit vaccine, which includes portions of CSP as the malaria antigen and is the first anti-malaria vaccine candidate ever to enter testing in phase III clinical trials in Africa. ${ }^{43,44}$

\section{Genetically Arrested Parasites (GAPs): Tailor-Made Vaccine Lines}

Near-complete Plasmodium genome sequence data, ${ }^{45}$ expression profiling, ${ }^{46}$ and reliable transfection technolog $y^{47}$ permit molecular genetics approaches to generate Plasmodium vaccine lines with precise gene deletions. ${ }^{14,48}$ Important requirements are (1) non-vital roles during asexual growth, where transfection is performed; (2) normal transmission, sporozoite formation inside the mosquito vector, and invasion of host hepatocytes; and (3) complete life cycle block between sporozoite entry and release of infectious merozoites from the liver. In a proof-of-concept study in the $P$. berghei/C57BL/6 model, targeted deletion of a signature liver stage protein, termed upregulated in infectious sporozoites gene 3 (UIS3), resulted in an early liver stage arrest and absence of blood stage infections, even when very high sporozoite doses were injected intravenously ${ }^{49}$ (Fig. 1). Immunization with three doses of uis3(-) sporozoites mounted long-lasting sterile protection against sporozoite re-infection, but not blood stage transfusion, supporting the notion that immunity is liver stagespecific. The concept to test genetically arrested parasites (GAPs) as experimental malaria vaccines was corroborated by additional targeted deletions of other liver stage-specific genes both in the P. berghei/C57BL/6 and the P. yoelii/Balb/c models. ${ }^{50-54}$ Protection offered following GAP-vaccination has been shown to be primarily dependent on CD8 T cells. ${ }^{53,55,56}$

Following the promising pre-clinical findings in rodent models, one vaccine line has been translated to $P$. falciparum by generation of $p 36 / p 36 p(-)$ parasites, despite the early indications of substantial break-through infections in the rodent model. ${ }^{57,58}$ It remains uncertain how this parasite line can be tested in human trials and highlights the need for rigorous pre-clinical testing.

In an effort to develop safe GAPs that display unconditional liver stage arrest a master regulator of gene expression in $P$. berghei sporozoites, termed SLARP (sporozoite and liver stage asparagine-rich protein), was targeted. ${ }^{59}$ Loss of SLARP function results in complete early liver stage arrest. When tested as an experimental malaria vaccine, slarp (-) sporozoites induced only modest and short-lived protection against reinfection. Expression profiling demonstrated that expression of a number of signature liver stage antigens, including UIS3 and UIS4, are controlled by SLARP, offering a molecular explanation for low vaccine efficacy of slarp(-) immunizations and opening a rationale for discovery of protective liver stage antigens by differential profiling. ${ }^{59}$ Experiments with the $P$. yoelii homologue, called sporozoite asparagine-rich protein 1 (SAP1), led to complete protection in the Balb/c malaria mouse model. ${ }^{60}$ This apparent discrepancy may be attributed to the presence of an immunodominant CSP $\mathrm{H} 2 \mathrm{~K}^{\mathrm{d}}$-restricted $\mathrm{CD}^{+}{ }^{+} \mathrm{T}$ cell epitope, ${ }^{61}$ corroborating the notion that the $P$. berghei/C57BL/6 model is the most difficult to protect against, and hence, serves as the most stringent preclinical model for anti-malaria vaccine development.

\section{Sporozoite Exposure during Drug Cover (SE/DC): A 'Needle'-Free Immunization Approach?}

The potential of treatment with registered antimalarial drugs during sporozoite exposure to induce potent immune responses was first tested in rodents. In these studies oral chloroquine (CQ) was given over a period of repeated sporozoite injections. ${ }^{62,63}$ Protection was also found to be mediated primarily by $\mathrm{CD}^{+}$ T cells. ${ }^{63}$ Roestenberg et al. could recently demonstrate the potency of this approach in human volunteers. ${ }^{64}$ In the vaccinees multifunctional (IFN $\gamma$ - and IL2-secreting) $\mathrm{T}$ cells of the effector memory phenotype $\left(\mathrm{CD} 62 \mathrm{~L}^{-}, \mathrm{CD}_{45 \mathrm{RO}^{+}}\right)$were consistently detected, but the precise molecular and immunological mechanisms of protection remain largely undefined. Individuals, who adhere to a strict CQ cover and effectively follow a SE/CQ scheme, can occasionally be found in malaria-endemic countries and were the basis for a successful antigen discovery program, which returned a number of liver stage antigens, e.g., LSA1. ${ }^{65}$ High anti-LSA1 antibody titers consistently correlate with a lower risk of clinical malaria. ${ }^{10,66}$ Although circumstantial, these findings support the premise that SE/CQ might have elicited liver stage immunity in the past. Because of widespread resistance of P. falciparum against $\mathrm{CQ}^{67}$ replacement drugs with CQ-like antiblood stage activity or, alternatively, with unconventional modes of action against the liver stage are essential for the translation of this concept into a clinical intervention.

The latter hypothesis was recently tested in rodent models using the liver-specific drug primaquine (PQ) as cover during sporozoite exposure $^{68}$ (Fig. 1). SE/PQ leads to a high degree of sterile protection after three rounds of immunization, including SE by bites of infected mosquitoes during the immunization phase, which most closely mimics natural transmission at night. Unfortunately, severe side effects of $P Q$ and other 8 -aminoquinolines limit the use of this intervention in malariaendemic countries. ${ }^{69}$ However, it will be interesting to test in a limited proof-of-concept clinical trail in humans whether SE/ PQ would also induce consistent protection against reinfection. 
If confirmed, the search for novel compounds that effectively kill intra-hepatic stages will gain further momentum.

More recently, studies in the $P$. berghei/C57BL/6 model established that concomitant use of antibiotics (Abx), such as azithromycin (AZ) and clindamycin, with infectious sporozoites does not lead to development of pathogenic blood stage infection despite full maturation of exoerythrocytic forms and release of hepatic merozoites. ${ }^{70}$ This so-called "delayed death" of parasites occurs after administration of antibiotics, which target the algae-originating apicoplast, an organelle encoding the prokaryotic-type translation machinery. ${ }^{71,72}$ When administered in a vaccination scheme, sporozoite exposure under Abx cover induces robust $\mathrm{CD} 8 \mathrm{~T}$-cell mediated protection against re-infection with mosquito-derived salivary gland sporozoites (Fig. 1). This strategy is apparently at least as powerful as the irradiated sporozoite gold-standard vaccine and might in fact be superior, since developmental arrest during late liver stage development may lead to improved live-attenuated vaccines due to the presence of a wider antigenic repertoire. Yet, correlates of protection, most likely protective $\mathrm{CD} 8{ }^{+} \mathrm{T}$-cell epitopes, remain to be identified. Systematic identification and characterization of these, still elusive signatures of protection will also inform the development of second-generation subunit vaccines.

Long-acting antibiotic drugs like AZ are being tested for mass drug administration, for instance in trachoma control programs. A recent documentation of an unanticipated substantial reduction in overall mortality after a campaign in Ethiopia ${ }^{73}$ encourages optimism that the protective effect observed in the experimental immunizations may be translated to malaria-endemic regions. One testable hypothesis is that intermittent delivery of AZ to the most vulnerable target populations, young children and pregnant women, may have a dual role in protection against malaria. In addition to an immediate therapeutic effect against an ongoing blood stage infection, sporozoites that are being delivered after bites of $P$. falciparum-infected mosquitoes will be arrested leading to gradual acquisition of anti-preerythrocytic immunity. Because of a potential vaccine-like secondary effect, oral Abx administration together with natural sporozoite exposure could effectively complement needle-vaccinations. Abx cover will also add an additional safety level or may even boost immunity in prospected experimental human trials with GAPs.

Due to its favorable drug safety record, AZ can be used in all ages and risk groups. ${ }^{74}$ The results of ongoing intermittent preventive treatment in pregnancy (IPTp) studies with a fixed drug combination azithromycin and chloroquine against uncomplicated malaria will inform future wider use and, perhaps, inclusion into other IPT programs.

\section{Outlook}

Development of a safe, affordable and long-lasting pediatric malaria vaccine and identification of immune correlates of protection among the abundant non-protective host responses remain research priorities in infection biology. Understanding the molecular and immunological mechanisms of the crosstalk between the parasite and the host is a prerequisite for rational anti-malaria vaccine discovery and development. Metabolically active, arrested liver stage parasites by live irradiation or molecular genetics and therapeutic cover during experimental sporozoite infections are alternative experimental whole organism vaccine strategies. Systematic immunological profiling of arrested parasites has the potential to inform translation of a whole organism anti-malaria vaccine to the human pathogen and can lead to the identification of protective antigens that have been elusive thus far. Together, precise genetic and pharmaceutical arrests of Plasmodium liver stage development are important approaches towards vaccine discovery.

\section{Acknowledgements}

We thank Diane Schad for the artwork. Work in our laboratories is supported by the Max Planck Society, Joachim Siebeneicher Foundation, the Chica and Heinz Schaller Foundation, and the European Commission (EviMalaR, \#34) to K.M., a University Research Fellowship from The Royal Society (UK) and a shortterm fellowship from the European Federation of Immunological Societies-Immunology Letters to J.C.H., the Deutsche Forschungsgemeinschaft (SFB 544, A7 and B10) to K.M. and S.B., and the Kenya Medical Research Institute/Wellcome Trust Research Programme, Kilifi to S.B.

\section{References}

1. Guinovart MM, Navia M, Tanner M, Alonso PL Malaria: burden of disease. Curr Mol Med 2006; 6:137-40; PMID: 16515506.

2. Haldar K, Murphy SC, Milner D Jr, Taylor TE. Malaria: mechanisms of erythrocytic infection and pathological correlates of severe disease. Annu Rev Pathol Mech Dis 2007; 2:217-49; PMID: 18039099; DOI: 10.1146/annurev.pathol.2.010506.091913.

3. Glynn JR, Bradley DJ. Inoculum size, incubation period and severity of malaria. Analysis of data from malaria therapy records. Parasitology 1995; 110:7-19; PMID: 7845714

4. Miller LH, Baruch DI, Marsh K, Doumbo OK. The pathogenic basis of malaria. Nature 2002; 415:673-9; PMID: 11832955; DOI:10.1038/415673a.

5. Hartgers FC, Yazdanbakhsh M. Co-infection of helminths and malaria: modulation of the immune responses to malaria. Parasite Immunol 2006; 2:497506; PMID: 16965285; DOI: 10.1111/j.13653024.2006.00901.x.
6. Bronzan RN, Taylor TE, Mwenechanya J, Tembo M, Kayira K, Bwanaisa L, et al. Bacteremia in Malawian children with severe malaria: prevalence, etiology, HIV coinfection and outcome. J Infect Dis 2007; 19:895904; PMID: 17299721.

7. Hill AV, Allsopp CE, Kwiatkowski D, Anstey NM, Twumasi P, Rowe PA, et al. Common west African HLA antigens are associated with protection from severe malaria. Nature 1991; 352:595-600; PMID: 1865923; DOI:10.1038/352595a0.

8. Marsh K, Kinyanjui S. Immune effector mechanism in malaria. Parasite Immunol 2006; 28:51-60; PMID: 16438676; DOI: 10.1111/j.1365-3024.2006.00808.x.

9. Langhorne J, Ndungu FM, Sponaas AM, Marsh K. Immunity to malaria: more questions than answers. Nat Immunol 2008; 9:725-32; PMID: 18563083; DOI:10.1038/ni.f.205

10. Crompton PD, Kayala MA, Traore B, Kayentao K Ongoiba A, Weiss GE, et al. A prospective analysis of the $\mathrm{Ab}$ responses to Plasmodium falciparum before and after a malaria season by protein microarray. Proc Nat Acad Sci USA 2010; 107:6958-63; PMID: 20351286; DOI: $10.1073 /$ pnas. 1001323107 .
11. Weiss GE, Traore B, Kayentao K, Ongoiba A, Doumbo S, Kone Y, et al. The Plasmodium falciparum-specific human memory B cell compartment expands gradually with repeated malaria infections. PLoS Pathog 2010; 6:1000912; PMID: 20502681; DOI:10.1371/journal. ppat.1000912.

12. Silvie O, Matuschewski K, Mota MM, Prudencio M. Interactions of the malaria parasite and its mammalian host. Curr Opin Microbiol 2008; 11:352-9; PMID: 18644249; DOI:10.1016/j.mib.2008.06.005.

13. Hafalla JC, Silvie O, Matuschewski K. Cell biology and immunology of malaria. Immun Rev 2011; In press.

14. Matuschewski K. Hitting malaria before it hurts: attenuated Plasmodium liver stages. Cell Mol Life Sci 2007; 64:3007-11; PMID: 17876525; DOI: $10.1007 /$ s00018-007-7263-z.

15. Richie TL, Saul A. Progress and challenges for malaria vaccines. Nature 2002; 415:694-701; PMID: 11832958; DOI:10.1038/415694a.

16. Malkin E, Dubovsky F, Moree M. Progress towards the development of malaria vaccines. Trends Parasitol 2006; 22:292-5; PMID: 16707275; DOI:10.1016/j. pt.2006.05.002. 
17. Matuschewski K. Vaccine development against malaria. Curr Opin Immunol 2006; 18:449-57; PMID: 16765576; DOI:10.1016/j.coi.2006.05.004.

18. Ballou WR, Arevalo-Herrera M, Carucci D, Richie TL, Corradin G, Diggs C, et al. Update on the clinical development of candidate malaria vaccines. Am J Trop Med Hyg 2004; 71:239-47; PMID: 15331843.

19. Freund J, Sommer HE, Walter AW. Immunization against malaria: vaccination of ducks with killed parasites incorporated with adjuvants. Science 1945 102:200-2; PMID: 17787139; DOI: 10.1126/ science.102.2643.200

20. Freund J, Thomson KJ, Sommer HE, Walter AW, Schenkein EL. Immunization of Rhesus monkeys against malarial infection (P. knowlesi). Science 1945; 102:202-4; PMID: 17787140; DOI: 10.1126/ science.102.2643.202.

21. Mulligan HW, Russell PF, Mohan BN. Active immunization of fowls against Plasmodium gallinaceum by injections of killed homologous sporozoites. J Mal Inst India 1941; 4:25-34.

22. Russell PF, Mulligan HW, Mohan BN. Active immunization of fowls against sporozoites but not trophozoites of Plasmodium gallinaceum by injections of homologous sporozoites. J Mal Inst Ind 1942; 4:311-9.

23. Richards WHG. Active immunization of chicks against Plasmodium gallinaceum by inactivated homologous sporozoites and erythrocytic parasites. Nature 1966; 212:1492-4; DOI:10.1038/2121492a0.

24. Nussenzweig RS, Vanderberg J, Most H, Orton C. Protective immunity produced by the injection of X-irradiated sporozoites of Plasmodium berghei. Nature 1967; 216:160-2; PMID: 6057225; DOI:10.1038/216160a0.

25. Vanderberg JP, Nussenzweig RS, Most H, Orton C. Protective immunity produced by the injection of $\mathrm{X}$-irradiated sporozoites of Plasmodium berghei. II Effects of radiation on sporozoites. J Parasitol 1968; 54:1175-80; PMID: 5757691.

26. Nussenzweig RS, Vanderberg JP, Most H, Orton C. Specificity of protective immunity produced by X-irradiated Plasmodium berghei sporozoites. Nature 1969; 222:488-9; PMID: 5768632; DOI:10.1038/216160a0

27. Nussenzweig R, Vanderberg J, Most H. Protective immunity produced by the injection of X-irradiated sporozoites of Plasmodium berghei. IV. Dose response, specificity and humoral immunity. Mil Med 1968; 134:1176-82; PMID: 4987036.

28. Vanderberg J, Nussenzweig R, Most H. Protective immunity produced by the injection of $\mathrm{X}$-irradiated sporozoites of Plasmodium berghei. V. In vitro effects of immune serum on sporozoites. Mil Med 1968; 134:1183-90; PMID: 4987037.

29. Vanderberg JP. Reflections on early malaria vaccine studies, the first successful human malaria vaccination and beyond. Vaccine 2009; 27:2-9; PMID: 18973784; DOI: 10.1016/j.vaccine.2008.10.028.

30. Nardin E, Nussenzweig RS. T cell responses to preerythrocytic stages of malaria: role in protection and vaccine development against pre-erythrocytic stages. Ann Rev Immunol 1993; 11:687-727; PMID: 8476576; DOI: 10.1146/annurev.iy.11.040193.003351.

31. Hafalla JC, Rai U, Morrot A, Bernal-Rubio D, Zavala $\mathrm{F}$, Rodriguez A. Priming of $\mathrm{CD}^{+} \mathrm{T}$ cell responses following immunization with heat-killed Plasmodium sporozoites. Eur J Immunol 2006; 36:1179-86; PubMed PMID: 16598821; DOI: 10.1002/eji.200535712.

32. Doolan DL, Hoffman SL. The complexity of protective immunity against liver-stage malaria. J Immunol 2000; 165:1453-62; PMID: 10903750.

33. Hafalla JC, Cockburn IA, Zavala F. Protective and pathogenic roles of $\mathrm{CD}^{+} \mathrm{T}$ cells during malaria infection. Parasite Immunol 2006; 28:15-24; PMID 16438672; DOI: 10.1111/j.1365-3024.2006.00777.x.
34. Overstreet MG, Cockburn IA, Chen YC, Zavala F. Protective CD8 T cells against Plasmodium liver stages: immunbiology of an 'unnatural' immune response. Immunol Rev 225:272-83; PMID: 18837788; DOI 10.1111/j.1600-065X.2008.00671.x.

35. Gwadz RW, Cochrane AH, Nussenzweig V, Nussenzweig RS. Preliminary studies on vaccination of rhesus monkeys with irradiated sporozoites of Plasmodium knowlesi and characterization of surface antigens of these parasites. Bull World Health Organ 1979; 57:165-73; PMID: 120766

36. Clyde DF, Most H, McCarthy VC, Vanderberg JP. Immunization of man against sporozoite-induced falciparum malaria. Am J Med Sci 1973; 266:169-77; PMID: 4583408.

37. Hoffman SL, Goh LM, Luke TC, Schneider I, Le TP, Doolan DL, et al. Protection of humans against malaria by immunization with radiation-attenuated Plasmodium falciparum sporozoites. J Infect Dis 2002; 185:115564; PMID: 11930326; DOI: 10.1086/339409.

38. Epstein JE, Rao S, Williams F, Freilich D, Luke T, Sedegah M, et al. Safety and clinical outcome of experimental challenge of human volunteers with Plasmodium falciparum-infected mosquitoes: an update. J Infect Dis 2007; 196:145-54; PMID: 17538895; DOI: $10.1086 / 518510$.

39. Ballou R. Obstacles to the development of a safe and effective attenuated pre-erythrocytic stage malaria vaccine. Microbes Infect 2007; 9:761-6; PMID 17412630; DOI:10.1016/j.micinf.2007.02.006.

40. Luke TC, Hoffman SL. Rationale and plans for developing a non-replicating, metabolically active, radiationattenuated Plasmodium falciparum sporozoite vaccine. J Exp Biol 2003; 206:3803-8; PMID: 14506215; DOI $10.1242 /$ jeb.00644

41. Hoffman SL, Billingsley PF, James E, Richman A, Loyevsky M, Li T, et al. Development of a metabolically active, non-replicating sporozoite vaccine to prevent Plasmodium falciparum malaria. Hum Vaccines 2010; 6:97-106; PMID: 19946222; DOI: 10.4161/ hv.6.1.10396.

42. Enea V, Ellis J, Zavala F, Arnot DE, Asavanich A, Masuda A, et al. DNA cloning of Plasmodium falciparum circumsporozoite gene: amino acid sequence of repetitive epitope. Science 1984; 225:628-30; PMID: 6204384.

43. Ballou WR. The development of the RTS,S malaria vaccine candidate: challenges and lessons. Parasite Immunol 2009; 31:492-500; PMID: 19691554; DOI: 10.1111/j.1365-3024.2009.01143.x.

44. Cohen J, Nussenzweig V, Vekemans J, Leach A. From the circumsporozoite protein to the RTS,S candidate vaccine. Hum Vaccines 2010; 6:90-6; PMID: 19806009; DOI: 10.4161/hv.6.1.9677.

45. Berry AE, Gardner MJ, Caspers GJ, Roos DS, Berriman M. Curation of the Plasmodium falciparum genome. Trends Parasitol 2004; 20:548-52; PMID 15522662; DOI:10.1016/j.pt.2004.09.003.

46. Tarun AS, Peng X, Dumpit RF, Ogata Y, Silva-Rivera $\mathrm{H}$, Camargo $\mathrm{N}$, et al. A combined transcriptome and proteome survey of malaria parasite liver stages. Proc Natl Acad Sci USA 2008; 105:305-10; PMID: 18172196; DOI: $10.1073 /$ pnas.0710780104.

47. Balu B, Adams JH. Advancements in transfection technologies for Plasmodium. Int J Parasitol 2007; 37:1-10; PMID: 17113093; DOI:10.1016/j.ijpara.2006.10.001

48. Vaughan A, Wang R, Kappe SHI. Genetically engineered, attenuated whole-cell vaccine approaches for malaria. Human Vaccines 2010; 6:107-13; PMID: 19838068; DOI: 10.4161/hv.6.1.9654.

49. Mueller AK, Labaied M, Kappe SHI, Matuschewski K. Genetically modified Plasmodium parasites as a protective experimental malaria vaccine. Nature 2005; 433:164-7; PMID: 15580261; DOI:10.1038/ nature 03188 .
50. Mueller AK, Camargo N, Kaiser K, Andorfer C, Frevert U, Matuschewski K, et al. Plasmodium liver stage developmental arrest by depletion of a protein at the parasite-host interface. Proc Natl Acad Sci USA 2005; 102:3022-7; PMID: 15699336; 10.1073/ pnas.0408442102.

51. Van Dijk MR, Douradinha B, Franke-Fayard B, Heussler V, van Dooren MW, van Schaijk B, et al. Genetically attenuated, P36p-deficient malarial sporozoites induce protective immunity and apoptosis of infected liver cells. Proc Natl Acad Sci USA 2005; 102:12194-9; PMID: 16103357.

52. Labaied M, Harupa A, Dumpit RF, Coppens I, Mikolajczak SA, Kappe SH. Plasmodium yoelii sporozoites with simultaneous deletion of P52 and P36 are completely attenuated and confer sterile immunity against infection. Infect Immun 2007; 75:3758-68; PMID: 17517871.

53. Tarun AS, Dumpit RF, Camargo N, Labaied M, Liu P, Takagi A, et al. Protracted sterile protection with Plasmodium yoelii pre-erythrocytic genetically attenuated parasite malaria vaccines is independent of significant liver-stage persistence and is mediated by CD8 ${ }^{+}$T cells. J Infect Dis 2007; 196:608-16; PMID: 1762484.

54. Falae A, Combe A, Amaladoss A, Carvalho T, Menard $\mathrm{R}$, Bhanot P. Role of Plasmodium berghei cGMP-dependent protein kinase in late liver stage development. J Biol Chem 2010; 285:3282-8; PMID: 19940133.

55. Mueller AK, Deckert M, Heiss K, Goetz K, Matuschewski K, Schlüter D. Genetically attenuated Plasmodium berghei liver stages persist and elicit sterile protection primarily via CD8 T cells. Am J. Pathol 2007; 171:107-15; PMID: 17591958.

56. Jobe O, Lumsden J, Mueller AK, Williams J, SilvaRivera H, Kappe SHI, et al. Genetically-attenuated Plasmodium berghei liver-stages induce sterile protracted protection that is mediated by MHC clas I-dependent IFN $\gamma$ producing CD8 ${ }^{+} \mathrm{T}$ cells. J Infect Dis 2007; 196:599-607; PMID: 17624847.

57. Van Schaijk BC, Janse CJ, van Gemert GJ, van Dijk MR, Gego A, Franetich JF, et al. Gene disruption of Plasmodium falciparum $\mathrm{p} 52$ results in attenuation of malaria liver stage development in cultured primary hepatocytes. PLoS One 2008; 3:3549; PMID: 18958160.

58. Van Buskirk KM, O’Neill MT, De La Vega P, Maier AG, Krzych U, Williams J, et al. Preeryhrocytic, liveattenuated Plasmodium falciparum vaccine candidates by design. Proc Natl Acad Sci USA 2009; 106:13004-9; PMID: 19625622.

59. Silvie O, Goetz K, Matuschewski K. A sporozoite asparagine-rich protein controls initiation of Plasmodium liver stage development. PLoS Pathog 2008; 4:1000086; PMID: 18551171.

60. Aly AS, Mikolajczak SA, Rivera HS, Camargo N, Jacobs-Lorena V, Labaied M, et al. Targeted deletion of SAP1 abolishes the expression of infectivity factors necessary for successful malaria parasite liver infection. Mol Microbiol 2008; 69:152-63; PMID: 18466298

61. Kumar KA, Sano G, Boscardin S, Nussenzweig RS, Nussenzweig MC, Zavala F, et al. The circumsporozoite protein is an immunodominant protective antigen in irradiated sporozoites. Nature 2006; 444:937-40; PMID: 17151604.

62. Beaudoin RL, Strome CPA, Mitchell F, Tubergen TA. Plasmodium berghei: immunization of mice against the ANKA strain using the unaltered sporozoite as an antigen. Exp Parasitol 1977; 42:1-5; PMID: 324783.

63. Belnoue E, Costa FTM, Frankenberg T, Vigario AM, Voza T, Leroy N, et al. Protective T cell immunity against malaria liver stage after vaccination with live sporozoites under chloroquine treatment. J Immunol 2004; 172:2487-95; PMID: 15470029. 
64. Roestenberg M, McCall M, Hopman J, Wiersma J, Luty AJ, van Gemert GJ, et al. Protection against malaria challenge by sporozoite inoculation. $\mathrm{N}$ Engl J Med 2009; 361:468-77; PMID: 19641203.

65. Guerin-Marchand C, Druilhe P, Galey B, Londono A, Patarapotikul J, Beaudoin RL, et al. A liver-stagespecific antigen of Plasmodium falciparum characterized by gene cloning. Nature 1987; 329:164-7; PMID: 3306406.

66. John CC, Tande AJ, Moormann AM, Sumba PO, Lanar DE, Min XM, et al. Antibodies to pre-erythrocytic Plasmodium falciparum antigens and risk of clinical malaria in Kenyan children. J Infect Dis 2008; 197:519-26; PMID: 18275273.

67. World Health Organization. Guidelines for the Treatment of Malaria. World Health Organization, Geneva 2006.

68. Putrianti ED, Silvie O, Kordes M, Borrmann S, Matuschewski K. Vaccine-like immunity against malaria by repeated causal-prophylactic treatment of liver-stage Plasmodium parasites. J Infect Dis 2009; 199:899-903; PMID: 19434915.
69. Lell B, Faucher JF, Missinou MA, Borrmann S, Dangelmaier O, Horton J, et al. Malaria chemoprophylaxis with tafenoquine: a randomised study. Lancet 2000; 355:2041-5; PMID: 10885356.

70. Friesen J, Silvie O, Putrianti ED, Hafalla JCR, Matuschewski K, Borrmann S. Natural immunization against malaria: causal prophylaxis with antibiotics. $\mathrm{Sci}$ Transl Med 2010; 2:49; PMID: 2063085.

71. Fichera ME, Roos DS. A plastid organelle as a drug target in apicomplexan parasites. Nature 1997; 390:407-9; PMID: 9389481

72. Sidhu AB, Sun Q, Nkrumah LJ, Dunne MW, Sacchettini JC, Fidock DA. In vitro efficacy, resistance selection and structural modeling studies implicate the malarial parasite apicoplast as the target of azithromycin. J Biol Chem 2007; 282:2494-504; PMID: 17110371
73. Porco TC, Gebre T, Ayele B, House J, Keenan J, Zhou $Z$, et al. Effect of mass distribution of azithromycin for trachoma control on overall mortality in Ethiopian children: A randomized trial. JAMA 2009; 302:962-8; PMID: 19724043.

74. Dunne MW, Singh N, Shukla M, Valecha N, Bhattacharyya PC, Dev V, et al. A multicenter study of azithromycin, alone and in combination with chloroquine, for the treatment of acute uncomplicated Plasmodium falciparum malaria in India. J Infect Dis 2005; 191:1582-8; PMID: 15838784

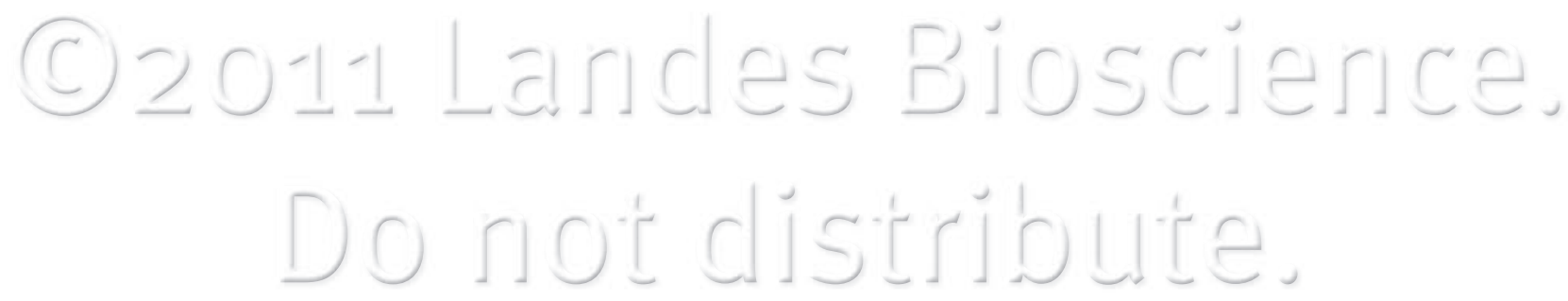

